\title{
Barère, penseur et acteur d'un premier opportunisme républicain face au directoire exécutif
}

\section{Pierre Serna}

\author{
(2) OpenEdition \\ Journals \\ Édition électronique \\ URL : https://journals.openedition.org/ahrf/828 \\ DOI : $10.4000 /$ ahrf.828 \\ ISSN : 1952-403X \\ Éditeur : \\ Armand Colin, Société des études robespierristes \\ Édition imprimée \\ Date de publication : 1 juin 2003 \\ Pagination : 101-128 \\ ISSN : 0003-4436

\section{Référence électronique} \\ Pierre Serna, «Barère, penseur et acteur d'un premier opportunisme républicain face au directoire \\ exécutif », Annales historiques de la Révolution française [En ligne], 332 | avril-juin 2003, mis en ligne le \\ 22 avril 2008, consulté le 24 avril 2022. URL : http://journals.openedition.org/ahrf/828 ; DOI : https:// \\ doi.org/10.4000/ahrf.828
}




\title{
BARÈRE, PENSEUR ET ACTEUR D'UN PREMIER OPPORTUNISME RÉPUBLICAIN FACE AU DIRECTOIRE EXÉCUTIF
}

\author{
PIERRE SERNA
}

\begin{abstract}
En l'an V, peu avant les élections de germinal, paraissent les réflexions de Barère, vivant alors en clandestinité, sur le gouvernement et le mode de fonctionnement du Directoire exécutif. Pour celui qui a été membre du Comité de salut public, au cceur de la Terreur, la République ne saurait se construire sans un gouvernement fort, doté d'un pouvoir exécutif puissant.

Cette préoccupation constitue un souci constant du député pyrénéen qui, en 1815, de nouveau représentant dans la chambre des Cent Jours, revient encore sur la nécessité d'une définition précise du pouvoir d'exécution. C'est justement cette constance qui permet de dépasser le débat sur les palinodies, prétendues ou réelles, de Barère, tout en pointant un premier opportunisme républicain. Le républicain intangible soutient une république "du juste milieu ", dont la caractéristique est un appareil exécutif fort, structuré, contrastant avec toute une génération de députés toujours méfiants vis-à-vis de l'Exécutif. Barère esquisse une république du centre et ce faisant s'inscrit dans un courant de pensée qui, de l'été 1791, à l'automne 1799, légitime l'utilisation brutale des prérogatives du pouvoir exécutif dans la perspective, toujours avancée et revendiquée, de sauver la Constitution ou la République. Ces hommes aux parcours divers, réunis enfin par Bonaparte, inventent ce que nous appelons la république de "l'extrême centre".
\end{abstract}

Mots clés : Barère ; pouvoir exécutif ; Directoire ; opportunisme républicain ; " extrême centre $n$.

Dans un article récent, Ran Halévi démonte, de façon fort convaincante, la mécanique du pouvoir exécutif au temps de la monarchie absolue (1). S'appuyant sur les modes de représentations du pouvoir du monarque, il montre comment, plus encore que par l'expression de l'auto-

(1) Ran HALEVI, "La Modération à l'épreuve de l'Absolutisme. De l'Ancien Régime à la Révolution ", Le Débat, mars-avril 2000.

Annales historiques de la Révolution française - 2003 - N 2 [101 à 128] 
rité du roi, c'est par l'intégration de la puissance du souverain sous forme de contrainte ou d'acceptation, que se produisait l'effectivité du commandement, sans que le roi n'ait véritablement à manifester son pouvoir authentique, ni à déchaîner ses foudres sur ses sujets. Le système ainsi analysé portait un nom : la monarchie tempérée... fruit d'une patiente construction de générations successives de juristes, depuis le XVIe siècle. Cette théorie se fondait sur la compréhension par le souverain, des lois fondamentales du royaume établissant ses devoirs et les limites de son autoritas, en même temps que, parallèlement, des règles non écrites, mais respectées, ébauchaient une esquisse des droits de chacun. À son tour, Georges Benrékassa a montré comment la modération, la tempérance, la raison, l'équilibre, le gouvernement au centre et du centre, à l'opposé des fanatismes, devaient animer le roi dans l'exercice impartial de son magistère (2). Tout ceci, comme le démontrent les deux historiens, présentait, dans le domaine de la philosophie politique et en théorie, un modèle parfait.

Une question demeure pourtant qu'ils n'ont pas manqué de poser : que se passe-t-il lorsque, de façon spontanée ou au contraire mûrement réfléchie, une résistance se manifeste, refusant le principe de l'obéissance au nom du seul respect dû à la chose décidée par le roi ? Dans ce cas, après la menace d'activer la foudre - et le Discours de la Flagellation le 3 mars 1766, est une expression manifeste - le roi est obligé de se voir contraint de dévoiler la face brutale que cachait la monarchie tempérée et à user de la force arbitraire pour imposer sa décision : il en va ainsi du coup d'État de Maupeou, lorsqu'un matin du mois de janvier 1771, les militaires vinrent signifier leur exil aux officiers du Parlement de Paris. Le réel et ses aléas brutaux, «despotiques», comme ne se privent pas de le souligner les officiers de justice du roi, victimes de l'arbitraire, fonctionnent ici comme les révélateurs d'une théorie politique de la tempérance (3). Cette remise en cause violente du pouvoir exécutif, qui empoisonne les rapports entre la monarchie et son autorité judiciaire, de 1750 à 1774, ainsi que la démonstration de force, censée la résoudre, entre 1766 et 1771 , a pour les deux acteurs - le gouvernant et le gouverné - des conséquences néfastes. Ni la monarchie atteinte dans son prestige, ni le Parlement blessé dans sa dignité, ne sortent victorieux d'une crise dont l'effet consiste à dévoiler le mécanisme répressif de l'institution royale, sans véritablement la consolider (4). Sans doute,

(2) George BenReKasSA, « Modération, Modérés, Modérantisme ", dans Rolf REICHARDT et HansJürgen LUSEBRINK, Hanbuch Politisch-Sozialer Grundbegriffe in Frankreich, 1680-1820, Munich, R. Oldenbourg Verlag, 1996.

(3) Sur la crise du pouvoir exécutif et le sens de l'autorité au XVIIIe siècle, cf. Hervé DREVILLON, « La Monarchie des Lumières : Réforme ou Utopie ? 1715-1774 », dans La Monarchie entre Renaissance et Révolution, 1715-1792, Joël CORNETTE (dir.), Paris, Seuil, 2000, pp. 284-354.

(4) William DoYLE, «The Parlements", dans Keith M. BAKER, The French Revolution and the Creation of Modem Political Culture, vol 1, The Political Culture of the Old Regime, Oxford, Pergamon Press, 1987. 
parce que l'un des modes de fonctionnement du pouvoir exécutif a toujours résidé dans ce halo de mystère, dans cette énigme que constitue la quantité de puissance réelle dont dispose le prince, et dont personne n'a jamais soulevé le voile. Or, une fois la manifestation de cette puissance mise en branle, les batteries s'étant dévoilées et leur portée finement appréciée, la crainte constitutive du rapport à l'autorité s'est estompée... les prétendus rebelles pouvaient continuer leur sédition de plus belle. Cette connaissance, désormais, des limites du pouvoir ne manque pas, en un moment où l'opinion apparaît - et c'est le cas pour les années 1770-1780 - de provoquer, au mieux une distanciation, un sérieux mépris au pire, qui s'emparent d'une couche de la population ne redoutant plus dorénavant les formes arbitraires de la volonté de l'Exécutif (5). Il devient donc normal, au début du règne de Louis XVI, que le débat se déplace sur la nécessaire transparence des institutions de commandement et porte sur l'exigence d'un éclairage des zones d'ombre du pouvoir. Comment est prise une décision? Comment sont mis en marche les leviers de l'application d'un décret royal? Désormais, les réformateurs n'ont de cesse que d'imaginer des formes de régénération de la monarchie, dans et par le contrôle de la décision royale, et donc, dans un premier temps, dans la stricte limitation du pouvoir exécutif, et ensuite, dans son empêchement à entretenir un secret, un mystère, jusque-là essentiels à son être.

Aussi quelques années plus tard les Assemblées de députés qui se succèdent de 1789 à 1795, construisent-elles un modèle politique posant clairement la prédominance du Législatif sur l'Exécutif. Durant l'été 1789, le pouvoir exécutif, associé à celui du roi, entaché de suspicion parce que rallié aux expressions autoritaires de la monarchie dite absolue, devient l'objet d'une réflexion intense dans l'ébauche, puis la rédaction progressive de la Constitution, au moins à deux moments clés : lors de la discussion du veto et lors du débat sur le mode de déclaration de la guerre, en mai 1790 (6). Ce discrédit profond du pouvoir exécutif s'est vu répété par de nombreuses générations d'historiens républicains, adoptant - consciemment ou pas - la même forme de rejet que leurs héros révolutionnaires. (7). Depuis quelques années, heureusement, cette carence historiographique a été fortement corrigée (8). Cette méfiance ne relevait pas seulement d'une posture idéolo-

(5) Roger CHARTIER, Les onigines culturelles de la Révolution française, Paris, Seuil, 1989.

(6) Marc BELISSA, Fraternité universelle et Intérêt national (1713-1795), Paris, Kimé, 1998, pp. 179-191, et Pierre SERNA, « Comment meurt une monarchie? 1774-1792 », dans Joël CoRNETTE, op. cit., « Mirabeau ou l'Hercule du pouvoir exécutif », pp. 406-409.

(7) D'Alphonse Aulard à François Furet, la posture intellectuelle offre de réelles similitudes : la séduction intellectuelle opère par-delà les décennies, présentant les orateurs et le débat parlementaire comme le cour de l'aventure révolutionnaire.

(8) Marcel MoRabito, Il Comando negato. Rivoluzione francese e Potere esecutivo, Manduria Piero Lacaita Editore, 1997, et Paolo Colombo, Govemo e Costituzione, la Trasformazione del Regime politico nelle Teorie dell'Età rivoluzionaria francese, Milano, Giuffré Editore, 1993. 
gique, incapable de saisir la richesse théorique d'une réflexion sur l'Exécutif. Elle traduisait à sa façon, le difficile rapport dans l'histoire des institutions républicaines, entre les pouvoirs législatif et exécutif, ce dernier trop souvent pris en flagrant délit d'outrepasser ses prérogatives, voire de se trouver à l'origine de coups d'État traumatisants pour l'idéal républicain : le 18 fructidor, le 18 brumaire, le 2 décembre 1851, sans compter au XXe siècle et ailleurs, la marche sur Rome, et plus près de nous, pour la génération qui nous a précédés, la naissance controversée de la Cinquième République et de sa constitution du «coup d'État permanent » et de l'immunité totale du chef de l'Exécutif (9)...

Un nouveau chantier de recherches, enfin, a stimulé une réflexion sur la question du pouvoir exécutif, en tentant de comprendre les motivations de tous ceux qui, agents du pouvoir exécutif ou victimes de ses décisions, avaient dû à plusieurs reprises, durant ces fameux vingt-cinq ans qui vont de l'été 1789 à l'été 1815, rendre compte de leur sinueux parcours (10). Une foule d'acteurs, anonymes parce que peu glorieux, en comparaison de leurs contemporains sous les armes, ou bien trop connus et vilipendés, parce que sans vergogne dans leurs revirements permanents, vont changer d'avis, d'attitudes, de postures. Sommés de s'expliquer sur leur comportement versatile par les gouvernements successifs ou par une opinion publique transformée en tribunal des consciences, ces protagonistes des États, royal agonisant, républicain naissant, impérial expirant ou monarchique restauré, avaient dû en permanence se justifier $a$ posterion, en construisant toujours des modes d'auto-légitimation de leur activité et de leur engagement, rendant compte, à leur avantage, cela va sans dire, de leurs responsabilités dans l'appareil exécutif de l'État. Ce faisant, cela n'empêchait pas les plus habiles ou les plus vils - c'est selon - d'essayer d'intégrer les nouvelles structures du pouvoir de l'Exécutif auxquelles ils se trouvaient confrontés, fussent-elles opposées à celles que ces «tourne-veste » avaient servi précédemment (11). Une recherche de l'adaptation ou du compromis permanent, une philosophie de l'intérêt personnel, élaborée en même temps que se produisent les bouleversements les plus profonds de la chose publique, symptômes graves, repérables dès la naissance de l'État républicain, marquent la trajectoire de bon nombre de ceux qui le servent (12). Cela n'empêchait pas non plus, d'autres hommes, honnêtes citoyens, d'interpréter leur parcours, dans la revendication d'une cohérence idéologique parfois réelle et sincère.

(9) Maurice Agulhon, Coup d'État et République, Paris, Presses de la Fondation nationale des sciences politiques, 1997.

(10) Cf. Politix, «Inconstances politiques », dossier coordonné par Brigitte GaITTI et Pierre SERNA, Volume 14, $n^{\circ} 56,2001$.

(11) Albert O. Hirschmann, Les Passions et les Intérêts, Paris, Hermès Sciences publications, 1997.

(12) M. Olivier BARUCH, Servir l'État français, l'Administration en France de 1940 à 1944, Paris, Fayard, 1997. 
N'était-ce pas là, l'expression d'une rigueur républicaine naissante qui revendiquait, pour son honneur, le service authentique de la respublica, service difficilement perceptible, et le plus souvent dénoncé comme agissant sous le travestissement calomnieux de la palinodie politique, à cause d'un déni facile et permanent de.toute justification sincère (13).

Se poserait donc la question du rapport entre le pouvoir exécutif propre à chacun des différents régimes et ces personnages, serviteurs de différents gouvernements, question éclairée de façon crue en ces lieux d'intersections des deux réalités hétérogènes, que sont les dates et crises de $1789,1792,1795,1797,1799,1804,1814$, mars et juillet 1815 . Face aux contingences de la réalité, à la nécessité de se frotter à une dimension particulière et quotidienne de l'administration ou de la politique, le comportement des agents de l'Exécutif, qui affrontent du fait même de leur statut, ces difficultés, et l'attitude de ceux qui ont reçu le surnom de "girouettes " - lorsqu'il ne s'agit pas des mêmes hommes - ne sont pas fondamentalement différents. Tous se trouvent confrontés à des situations objectives qui les dépassent le plus souvent et qu'ils doivent assumer de la manière la plus concrète. Si l'on y ajoute les perspectives de carrières politiques nouvellement apparues et l'appréhension des nouveaux habitus des fonctionnaires, qui n'ont pas manqué de s'imposer aux témoins, participants et survivants des 9 Thermidor et 18 Brumaire, c'est-à-dire à l'écrasante majorité d'une classe politique s'essayant, depuis 1789 , à redéfinir les différents pouvoirs, alors devient bien plus pertinente la question de la versatilité des opinions et des ralliements qu'elle provoque, conjuguée à celle de la conquête de ces places qui, dans l'appareil d'État - administration ou poste de décision sont proches du pouvoir exécutant (14). L'idée d'un lien entre la "versatilité " répétitive, non négligeable, du milieu politique d'une part, et la construction d'un nouvel appareil exécutif de l'autre, évoque non pas seulement la juxtaposition de deux phénomènes, que le hasard de l'événement, ou la seule nécessité de la conjoncture, relient entre eux, mais force à penser le nœud qui unit deux pratiques. L'hypothèse de travail peut être formulée ainsi : l'élaboration de l'Exécutif pendant les vingt-cinq ans qui nous intéressent, et les politiques de ralliement, pendant et après ce quart de siècle, même si elles apparaissent $a$ priori distinctes, présentent néanmoins des points de convergence notables, bien que longtemps passés sous silence ou demeurés inaperçus.

Pourquoi? Revient en mémoire la leçon politique de cette monarchie, tempérée en théorie, mais qui se traduisait infailliblement en monarchie

(13) Sur la naissance de cette culture de l'État, dans la construction des habitus de fonctionnaires, par exemple, cf. KAWA, Ronds de Cuir en révolution; étude prosopographique des employés du ministère de l'Intérieur sous la Première République (1792-1800), Paris, Éd. du CTHS, 1996.

(14) Sur la professionnalisation des révolutionnaires, cf. Bronislaw BACZKo, Comment sortir de la Terreur, Thermidor et la Révolution, Paris, Nrf-Gallimard, 1989, « Le Moment thermidorien », pp. 305-350. 
absolue et arbitraire dans la réalité des faits. L'observation des régimes qui se succèdent de 1789 jusqu'à 1815 , la fréquentation de ceux qui détiennent le pouvoir exécutif ou en sont l'expression incarnée, de Robespierre à Bonaparte, en passant par les cinq Directeurs, sans oublier le Barnave de l'été 1791, montrent que tous n'ont eu de cesse que de rappeler leur volonté de piloter la barque de l'État entre les deux écueils de l'ultra et de la ContreRévolution. À partir de positions évidemment différentes, les uns et les autres n'ont eu d'autre volonté que de construire ou d'instrumentaliser les formes de radicalisation de la droite et de la gauche, afin de caler le timon du pouvoir exécutif en un centre, synonyme de possibilisme, si on le compare aux programmes de ses opposants. Ce centre s'avère omniprésent et éminemment réactif aux deux pôles d'émissions idéologiques rouges et blanches, qui l'encadrent, et demeure néanmoins, une entité difficile à cerner, parce qu'échappant sans cesse aux cadres de la discussion idéologique, et revendiquant la pratique avant tout comme valeur fondatrice (15). Comme ce fut le cas pour la monarchie, cette théorie centriste du pouvoir, cette occupation de force, et en même temps forcée, d'un centre contraignant les oppositions décrétées à droite et à gauche à la radicalisation, se manifeste encore et sans exception, dans la contingence du moment et le réel des situations, par un arbitraire et une rigueur absolue dans l'exercice de la loi martiale d'abord, que ce soit sous la Terreur ensuite, sous les coups de force du Directoire ou sous les lois liberticides du Consulat et de l'Empire, pour finir.

N'est-ce pas là - encore une hypothèse de travail - que s'élabore une idéologie sans discours politique, une opinion du pouvoir exécutif, sans configuration exacte, à l'opposé des « factions " qu'elle ne cesse de dénoncer, donc insaisissable? Appelons-la l'« extrême centre »! Elle s'avère non moins radicale que les forces centrifuges qu'elle dénonce, et bien mieux embusquée parce qu'elle calque sa position mimétique sur les transformations de l'Exécutif, d'une expression républicaine à l'autre. Authentique violence faite au débat démocratique, cet «extrême centre », issu d'une pratique de l'appareil exécutif, et sans cesse présenté et traduit comme étant la défense des honnêtes ou des vertueux, ne se présente pas de façon monolithique. Tout comme la Contre-Révolution et l'aile jacobine dans leurs multiples différences, il offre également ses nuances, ses infinies variations, voire ses oppositions internes, voire encore ses contradictions nées de l'exercice du pouvoir.

Ces remarques liminaires interdisent de simplifier par trop l'hypothèse de travail, ou de prêter quelque peu naïvement le flanc à une critique de la part de tous ceux qui seraient choqués par le néologisme « extrême centre»,

(15) Christine LE BOZEC, « En sortir par le centre ", dans La Révolution française, idéaux, singularité, influences (...) dans Robert CHAGNY et Alain CHEvaliER (dir.), Grenoble, Presses Universitaires de Grenoble, 2002, pp. 359-366. 
trouvant le terme vague - il ne peut être ontologiquement que cela - ne voyant là qu'une proposition masquée, visant à réduire le débat par une comparaison entre absolutisme royal, terreur absolue, et empire absolument policier, faisant semblant de croire que l'on voudrait faire l'amalgame entre Louis XIV, Robespierre et Napoléon ou encore limiter la richesse et diversité du débat révolutionnaire à de simples enjeux de conquêtes et de jouissance du pouvoir (16).

\section{Barère, un cas d'école}

Ici, se dresse un personnage qui tient lieu de paradigme aux premiers pas de cette recherche. Il permet de réunir les données préalables de l'expérience, en présentant dans son parcours biographique une existence qui réunit les trois éléments exogènes à faire fusionner, d'abord une pratique du pouvoir exécutif, ensuite un parcours dénoncé de la façon la plus violente sous les traits du Judas politique, enfin une réflexion sur la nécessité de construire une république du centre qu'il appelle « le pouvoir du juste milieu ».

Bertrand Barère de Vieuzac est cet homme, authentique républicain, indéfectiblement choisi par ses électeurs des Hautes-Pyrénées, de 1789 à 1815 , et encore après son retour d'exil, en 1830 , jusqu'à son décès en janvier 1841. Il fut un acteur de tout premier plan de la Terreur, puisque membre du Comité de salut public, puis penseur de l'organisation du pouvoir exécutif dans sa retraite forcée, sous le Directoire, et de nouveau sur le devant de la scène durant les Cent Jours, non sans avoir été, au début du Consulat sollicité ou avoir sollicité, lui-même, les faveurs du général Bonaparte, en rédigeant pour ce dernier des notes ou encore un journal virulent contre la perfide Albion (17). Barère offre le parcours d'un praticien et, en même temps, d'un théoricien du pouvoir exécutif. Il est l'image possible d'un expert de la machine gouvernementale et de son mode de fonctionnement central. Il semble incarner à la fois, la figure d'un premier radicalisme et d'un opportunisme républicain.

(16) Précisons que cette notion opératoire « d'extrême centre " doit être saisie, avec prudence, tel un outil, permettant de proposer un terme afin de circonscrire, s'il se peut, une réalité non nommée par les contemporains et pourtant efficiente. Il n'y a pas ici prétention à inventer une catégorie politique nouvelle ni à l'imposer en tant que telle. Les deux termes accolés n'ont pas pour fonction de cataloguer ou d'enserrer sous une étiquette politique rigide des personnes aux parcours politiques différents, mais ont pour vocation d'aider à décrypter des postures et des convictions qui, pour ne pas s'énoncer sous la forme d'un désignant collectif, n'en ont pas moins existé.

(17) Sur Barère, voir parmi les textes publiés, L GERSHOY, Bertrand Barère, a Reluctant Terrorist, 1962 ; J.-P. Thomas, Bertrand Barère. La Voix de la Révolution, Paris, Desjonquères, 1989; également l'article de Françoise BRUNEL, «Les Institutions républicaines : Projet démocratique, Horizon d'Attente et/ou Utopie (an II-an V) ", dans La Révolution française, idéaux singularités (...), op. cit., pp. 319-328, et K. YAMAZAKI, "Les éloges de Montesquieu par Barère », Study Series, $n^{\circ} 18$, Center for Historical Science Literature, Hitotsubashi University, mars 1989,49 p. 
Il est revenu à Macaulay, outré par l'anglophobie de Barère, de se proposer comme le principal artisan de la légende noire du personnage, chargé de tous les vices imaginables. Vil et versatile, c'est l'inconsistance qui caractériserait l'homme.

«Barère entra dans la vie politique, sans dessein, ni opinion arrêtés; esclave du dernier mouvement, royaliste, aristocrate, démocrate, suivant l'avis qui prévaut dans le café ou le salon où il se trouve en ce moment même [...] Royaliste ou girondin, jacobin ou impérialiste, il demeura toujours trissotin [...] Au retour des Bourbons, il redevint royaliste [...] Cette apostasie ne lui apporta rien, par même l'addition d'une infamie nouvelle : son caractère était par trop noir pour qu'il pût le noircir encore». Il devient député pendant les Cent Jours dans l'« assemblée la plus absurde qui se fût jamais réunie en France »(18). Point trop dupe de son entourage et déjà fort conscient de l'épouvantable réputation qui le précède, Barère, celui qu'on a déjà surnommé l'«Anacréon de la guillotine » a fort lucidement cerné le problème qui le concerne dans la volonté de ses contemporains de le cataloguer chaque fois du côté des plus forts, au gré des circonstances (19). Personnage caméléon, il est accusé de s'être glissé sous plusieurs déguisements idéologiques... Lui-même dans ses Mémoires connaît bien cette épouvantable réputation et dresse la liste des appartenances partisanes réelles ou supposées que ces proscripteurs lui ont prêtées, dans le catalogue de l'arlequinade politique : il aurait été successivement jacobin, feuillant, girondin, terroriste, robespierriste, anarchiste, républicain, bonapartiste... (20).

La question du pouvoir exécutif républicain permet d'aborder, tout autrement qu'il n'a été présenté jusque-là, le problème des volte-face déconcertantes de Barère. La versatilité du personnage ne devrait pas être mesurée à l'aune des changements intéressés, dans un débat idéologique, étalé sur plus d'un quart de siècle, et dont il est possible d'imaginer, à moins d'avoir une conception quelque peu rigide de la droiture politique, qu'il évolue souvent en fonction des modalités d'expressions différentes, sans que les champs d'horizon ne varient. Vouloir défendre Barère de la critique de «vil gascon » revient finalement à entrer dans le jeu de ses détracteurs (21). En revanche, ce qui est examiné dans les pages qui suivent, ce sont les diffé-

(18) MACAULAY, Bertrand Barère, traduit de l'anglais par Édouard Gibert, Paris, Dentu, 1888, Essai traduit en 1844, p. 29.

(19) Voir Bertrand BARÈre, Mémoires de Bertrand Barère, Membre de la Constituante, du Comité de salut public, et de la Chambre des représentants, publiés par Hippolyte Carnot, et David (d'Angers), Paris, chez Jacques Laffite, 1842, BNF M 10765. Cf. aussi le commentaire que fait Sergio LuZzATo sur la fiabilité du texte, dans $l l$ Terrore ricordato, memoria e tradizione dell'esperienza rivoluzionaria, Genova, Marietti, 1988, trad. fr. Lyon 1995. Sur la logique pro domo des acteurs de la Terreur, durant la Restauration, voir Michel BIARD, Missionnaires de la République. Les représentants du peuple en mission (1793-1795), Paris, Éd. du CTHS, 2002, chap. VII « Du banc des accusés à la damnatio memoriae », pp. 339-396.

(20) B. BARÈRE, Mémoires, op. cit, tome II, p. 102.

(21) Maïté BOUYSSY, « Barère, vil Gascon, un Élément Écran de l'Historiographie », Lengas, n 34 , 1993, pp. 69-109. 
rentes positions de Barère, non dans la discussion politique, mais face à la faisabilité pratique du gouvernement républicain. En ce sens, les attitudes changeantes de Barère ne renverraient pas tant à une faiblesse idéologique, qu'à une force d'expérimentation permanente de l'essence des modes d'application de la loi républicaine sous différents gouvernements. Ainsi les positions diverses de Barère se résumeraient à quelques questions non résolues jusque dans les années 1880 : qu'est-ce qu'un pouvoir exécutif républicain? peut-il se construire ailleurs que dans ce même rapport de contrainte idéologique qui a transformé l'absolutisme externe de la monarchie en une auto-contrainte interne des institutions républicaines? peut-il se construire autrement que dans la marginalisation continuelle des forces radicales de gauche ou de droite? comment construire un Exécutif qui puisse garantir que l'application de la loi républicaine n'est pas seulement le résultat de l'intégration par les citoyens, d'une obéissance passive, indifférente, mais la condition de l'augmentation de leur liberté ?

En réalité, même si le personnage a semblé offrir, au gré des circonstances et des transformations violentes de gouvernement ou de régime, tous les stigmates du reniement, il n'en apparaît pas moins que du journaliste du Point du Jour qui commente les conditions d'organisation du veto, premier grand débat constitutionnel sur le pouvoir exécutif, en 1789, jusqu'à l'homme vieilli qui prend la parole le 5 juillet 1815 , encore sur la question du veto et de la sanction de la loi, cette fois conjointement partagée par le monarque et les assemblées, une vraie continuité et une réelle cohérence de pensée s'imposent, au moins sur ce thème. Entre le porte-parole du Comité de salut public qui réfléchit sur la notion d'esprit public et le clandestin sans papiers, proscrit du Directoire, qui, en l'an V, disserte sur Montesquieu et la construction d'un esprit public dans une république ancienne, l'affinité est grande. Dans son immense bibliographie, Barère n'a cessé de produire des textes sur le pouvoir exécutif, fasciné qu'il était par ce côté obscur de la chose républicaine. Très tôt si l'on en croit ses Mémoires, largement corroborés par les débats d'assemblée, Barère a insisté sur la nécessité d'élaborer, sans crainte, un pouvoir exécutif fort : "Il ne faut pas s'abuser : en matière d'organisation des pouvoirs politiques, le pouvoir exécutif a, de sa nature, une telle action que, s'il ne se trouve pas suffisamment doté, il ne cherche qu'à étendre sa prérogative; si vous le rendez impuissant, il devient usurpateur » (22). Par la suite, assumant son contre-pied par rapport à une pensée dominante toujours inquiète des éventuels débordements du pouvoir de l'action, pour reprendre une catégorie développée par Mirabeau, il n'a cessé d'en proposer une définition originale, sous la forme d'une pensée du gouvernement global, doté de larges prérogatives. De plus, alors que les 
rares contemporains qui ont médité les conditions effectives du pouvoir exécutif, ont le plus souvent conçu la condition de son contrôle en proposant soit une fragmentation des pouvoirs, soit une stricte séparation de ceux-ci, comme le tente en vain la Constitution de l'an III, soit une forme de pouvoir neutre, au-dessus des deux autres pouvoirs, théorie dont Sieyès s'est fait le grand avocat, c'est au contraire, au-dessous de la structure gouvernementale, dans les forces vives et la construction de l'opinion, parties prenantes d'un Exécutif sans cesse pensé en fonction de sa représentativité, que se trouve l'apport novateur de Barère et sa constance dans la démonstration, à deux moments clés de grande crise politique.

C'est tout d'abord, durant le Directoire et plus particulièrement en l'an $\mathrm{V}$, peu avant les élections qui donnent la victoire aux partisans d'une monarchie constitutionnelle et font basculer le premier Directoire dans la pratique du coup d'État légal, puis durant les Cent jours et l'essai improbable d'une forme constitutionnelle, entre le 3 juin et le 8 juillet 1815 , que Barère développe deux points de vue où il ne manque pas de perspicacité et de fidélité à son idéal républicain d'exécution et de recherche des meilleures conditions d'application de la loi.

\section{Le pouvoir exécutif redéfini. Barère et les Cent Jours.}

Il était une fois, à Orléans, un brave abbé fort inquiet des désordres causés par le retour impromptu de Napoléon de l'île d'Elbe et sa remontée triomphale sur Paris (23). Le pauvre homme, comme bon nombre de personnes dans cette France timorée ne sachant plus à quel chef se vouer, était encore plus soucieux à l'idée de la boîte de Pandore ouverte quelques jours avant la funeste bataille du Mont-Saint-Jean, par l'inauguration des travaux parlementaires de l'assemblée élue au mois de mai 1815 et chargée d'appliquer l'acte additionnel aux Constitutions, transformant l'empire autoritaire en empire libéral constitutionnel (24). Parmi les députés, on retrouvait des hommes, longtemps muselés par la police de Fouché mais qui n'avaient rien oublié de leur vieil idéal républicain et dont les noms seuls suffisaient à épouvanter le curé de province. Un élu du département des Hautes-Pyrénées, tout particulièrement, rappelait la Terreur, de sinistre mémoire et sur laquelle on a tant écrit. Un an après l'aventure des Cent Jours, la mémoire était vive encore et l'angoisse réelle d'avoir vu les hommes de 1793 , toujours à pied d'œuvre, revenus dans les fourgons de

(23) PATAUD, abbé de, Mémoires et joumal tenus de 1796 à 1816, ceuvres diverses et confessions précédées d'une table par Vergnaud Romagnési, Orléans, 1796-1816, 15 vol. dans 16. A.D. Loiret, p. 1776.

(24) Voir Eugénio di RIENzo, L'Aquila e il Berretto frigio, per una Storia del Movimento democratico in Francia da Brumaio ai Cento Giomi, Napoli, Edizioni scientifiche Italiane, 2001. 
l'empereur. «Je détestais Bonaparte parce qu'il est dans mon caractère de détester les despotes, mais je craignais le retour d'une république présidée par Carnot, Foucher [sic] de Nantes, Barrère et autres jacobins » (25). Durant les Cent Jours, la position de Barère au cours de l'été 1815, lorsqu'il est encore une fois choisi par ses concitoyens des Hautes-Pyrénées pour les représenter, ne manque pas d'intérêt car elle concerne presque exclusivement des aspects du pouvoir exécutif et les façons de réguler les rapports de celui-ci avec les autres pouvoirs. Les sept interventions de Barère pendant ces mois de juin et juillet, portent sur des aspects essentiels de construction et de régulation du pouvoir exécutif et, par certains côtés, retentissent comme un bilan de sa réflexion.

Sa première intervention, le 16 juin, développe les modalités de communication entre l'Assemblée et le pouvoir exécutif. Authentique mémoire des assemblées de la Révolution, Barère rappelle, en faisant référence à 1789,1792 et 1795 , que les ministres doivent se déplacer en personne afin d'engager leur responsabilité personnelle devant les députés (26). Le lendemain, il revient sur ce thème de façon différente : "Votre Constitution a donc deux sortes d'initiatives, l'une de la part du gouvernement, l'autre de la part des Chambres; ainsi nous voilà revenus au véritable système représentatif; or, dans tous les geuvernements représentatifs, c'est le gouvernement qui présente le tableau de la situation, et c'est la législature qui indique les éléments dont il faut se servir et les moyens qui sont à prendre » (27).

Du point de vue du vocabulaire, il est intéressant de constater que le mot gouvernement désigne deux choses différentes : il est le gouvernement (groupe de ministres), mais il est également synonyme de système représentatif. Ce qui pourrait passer pour une confusion, révèle, en fait, la clé de voûte du système de Barère. Linitiative constitutionnelle dont peuvent s'emparer les députés fait partie du «gouvernement ». Le pouvoir exécutif relèverait d'une responsabilité collective englobant les députés et permettant l'initiative conjointe de la proposition de loi. Il en va de la légitimité retrouvée et républicaine du pouvoir exécutif. Concrètement, en ce 17 juin 1815, Barère appelle à la constitution « d'un pouvoir extraordinaire : c'est la dictature de la loi qu'il faut établir, et non celle d'un homme. Vous pouvez l'établir pour un temps limité, dans des termes dignes d'un peuple libre, et vous vous y refuseriez ! $\gg(28)$

(25) Abbé Pataud, op. cit.

(26) Le Moniteur, 16 juin 1815, p. 461.

(27) Id., 17 juin 1815 , p. 477.

(28) Il faut que la critique contre Barère soit à son tour cohérente. Vingt-deux ans après la Terreur, on peut encore lui reprocher son réflexe terroriste d'occupation légale des prérogatives de l'Exécutif par une Assemblée constituante, même en temps de crise politique; mais il devient alors plus difficile de dénoncer ses palinodies. 
Le 5 juillet, alors que la vacance du pouvoir met en danger le pays, Barère intervient sur la notion de sanction de la loi, essentielle selon lui, car elle crée de fait, une réciprocité de contrôle entre le pouvoir législatif et le pouvoir exécutif, tous deux dotés de la faculté de proposition et de la faculté de sanction. Le lendemain, au moment de débattre sur l'article 10 relatif à la définition du gouvernement et à la division des pouvoirs, alors que le représentant Flaugergue défend le principe que seuls les députés sont la représentation nationale et, à ce titre, votent souverainement l'impôt, un autre membre fait remarquer que le monarque, tout comme la Chambre des pairs ou les représentants, détient le caractère de la représentation. Barère intervient alors de façon décisive. Il demande que le «mot de représentation nationale soit consacré ; c'est le lien [dit-il], le nodus de tous les pouvoirs. En Angleterre, le grand représentant se compose du roi, des pairs, des communes. Personne n'est représentant, mais les trois pouvoirs composent la représentation nationale. Le prince est inamovible, les pairs sont un pouvoir médiat, les représentants un pouvoir immédiat. Ce sont ces principes qu'il importe de consacrer, ceux auxquels l'Angleterre doit la stabilité de son gouvernement ». Barère, par touches quasi quotidiennes, complète et enrichit sa conception du pouvoir. Ici, la globalité et la coresponsabilité de la représentation par des institutions diverses et de nature différente, forment un ensemble insécable et complexe (modelé sur l'ennemi de toujours), mais assurent enfin l'effectivité du gouvernement, c'est-à-dire sa stabilité et, souci toujours majeur, l'effectivité de l'application et de l'obéissance à la loi. Il n'est pas sans intérêt de constater que le chapitre troisième de la Constitution, discuté dans les moments qui suivent, définit le gouvernement de la France, au moment où, dans l'assemblée, des voix s'élèvent pour que le roi prête serment de maintenir, non pas un nouveau texte, mais la Constitution tellement vilipendée et soi-disant inviable de l'an III.

Le 7 juillet, Barère prend une dernière fois la parole, à propos de la composition de la Chambre des pairs dont le rôle doit être d'assumer l'équilibre entre le gouvernement et la Chambre des représentants, et donc d'assurer le contrôle et le bon fonctionnement du pouvoir exécutif. «Persuadé qu'à l'exemple d'un ancien législateur, il faut composer avec les circonstances et donner aux Français les lois que comportent leurs mœurs et même leurs préjugés, [il] propose d'établir que les membres de la Chambre des pairs seront nommés à vie par le monarque "; encore une fois, pour qui connaît Barère, la cohérence de sa conception du pouvoir apparaît entre les lignes. Circonstances et préjugés sont des réalités contre lesquelles le plus républicain des législateurs ne peut aller, à moins de n'accorder aucune importance aux effets de réel, induits, plus que par le pouvoir exécutif, par la prégnance et la gravité des événements, qu'un homme politique responsable aurait du mal à négliger en ce mois de juillet 1815 , début de grande terreur 
blanche. Barère, et ce malgré l'adversité des faits, continue à penser la régulation et l'effectivité du pouvoir exécutif comme indissociables.

Ces prises de parole publiques et politiques montrent d'abord l'intensité du débat, alors que les étrangers sont dans Paris et menacent en permanence les députés des Cent Jours. Elles montrent aussi combien demeurent importants dans la construction et la tenue du débat, les hommes qui ont été les inspirateurs de la Constitution de 1795, Boissy d'Anglas, Lanjuinais, Garat, authentiques artisans de la sortie constitutionnelle des crises politiques que traverse périodiquement la France. Concernant Barère, ses prises de position ont été préparées par une série d'écrits qui, depuis le début des Cent Jours, ont signifié une reprise de ses réflexions sur la nature du pouvoir à proposer à la France. Trois documents, rédigés entre avril et mai 1815, posent des jalons permettant de comprendre la nature de ses interventions au mois de juillet suivant: la théorie de la Constitution de la Grande-Bretagne, les considérations sur la chambre des Pairs, et finalement, les commentaires sur les époques de la nation française et les quatre dynasties, avaient déjà placé la construction du pouvoir exécutif au cœur de ses réflexions (29).

Dans son dernier essai et de façon saisissante, dressant à la manière de l'époque, en quelques pages synthétiques, un tableau de l'histoire de France, depuis Clovis jusqu'à l'actualité du printemps 1815, Barère évoque les différents pouvoirs qui se sont succédés. Finalement, l'histoire politique de ce pays n'est qu'une longue crise du pouvoir exécutif, entrecoupée de quelques périodes plus sereines qui ont toujours coïncidé avec la construction d'un gouvernement stable, élaborant une légitimité incontestée, et uniquement conférée par l'élargissement et l'intégration de la souveraineté des communes, du peuple ou du tiers, selon les siècles examinés. Des Gaulois réunis dans l'assemblée annuelle du Champ de Mars, à la nation convoquée par le roi en 1789 , en passant par l'intégration du peuple dans l'administration, qu'avait désirée Philippe le Bel, le seul « secret » de l'Exécutif se trouve là, dans le consentement mutuel de son fonctionnement. Hors de ces quelques périodes, l'Histoire est bien celle de la crise permanente de l'Exécutif. «Ainsi, en se résumant, les Français ont eu dix siècles d'aristocratie féodale, sacerdotale, nobiliaire, trois siècles de despotisme royal, ministériel et parlementaire, quarante ans de guerres civiles sous les Valois, cinquante ans de dissensions civiles, de troubles religieux sous les Bourbons,

(29) B. BARÈRE DE VIEUZAC, député des communes aux États généraux, à l'Assemblée constituante et du peuple français, à la Convention nationale, membre du collège électoral du département des Hautes Pyrénées, Théorie de la Constitution de la Grande Bretagne ou de ses trois Pouvoirs séparés et réunis, ouvrage traduit de l'Anglais de Brooke, précédé d'un avertissement du traducteur et d'un examen rapide des Constitutions qui se sont succédés en France depuis 1791 jusqu'en 1814, avril 1815, chez Louis Colas, à Paris; Considérations sur la Chambre des Pairs, ou sur la Chambre des Représentants héréditaires avant qu'elle soit acceptée, établie et composée, à Paris, Chez Delaunay, 20 mai 1815; Les Époques de la Nation française et les quatre Dynasties, à Paris, chez L. Colas, 23 mai 1815. 
vingt-cinq années de changements politiques, de guerres civiles et étrangères, pendant la Révolution, et il n'y a pour la nation, pour la démocratie, que les six années qui se sont écoulées depuis 1789 à 1794 . Il n'y a eu de suspension pour les diverses aristocraties, que depuis la Constitution de 1791 jusqu'à la Constitution de 1804 » (30).

La fin du texte souligne l'importance de l'expérience d'un empire libéral. «Le glorieux fondateur de la quatrième dynastie est rappelé par les vœux du peuple et par les souvenirs de l'armée, la souveraineté de la nation est de nouveau proclamée et reconnue [...] Avec une bonne et sage Constitution, avec un prince légitimé par la volonté nationale, la France souveraine, est inviolable dans ses libertés comme dans son territoire; avec ses soldats citoyens, avec ses valeureuses armées et un prince plein de génie et de gloire, la France libre est invincible » (31). Quelques semaines auparavant, Barère s'était contenté d'une rétrospective historique plus modeste, circonscrite à l'étude des Constitutions que la France s'était données depuis 1791, en les comparant à la stabilité du modèle anglais à partir de la traduction du texte anglais de Brooke datant de 1769, Théorie de la Constitution de la Grande-Bretagne. Lintroduction pose de façon claire les enjeux du débat. La Constitution se présente telle une mécanique fondée sur la division des pouvoirs, qui fonctionnent, eux, au moyen de balanciers, de contre-poids, d'énergie organisée, capables de faire mouvoir les principes aristocratiques, royaux et démocratiques, afin de les réunir enfin dans un faisceau, le "système représentatif anglais ", dont les cent vingt-cinq ans de marche constituent le meilleur avocat. Deux idées-forces s'imposent. La politique n'est pas un corps, elle n'est pas un organe. Elle est une science de la mécanique, une construction physique dont la vocation englobante est de faire du gouvernement une image de tous les régimes. Dans cet ouvrage, le pouvoir synthétise celui de tous les gouvernements. C'est d'ailleurs de la complexité de cette réunion et de son champ d'application étendu que dépend le bon gouvernement et non, comme on pourrait le croire, des conditions d'étroitesse du pays ou de l'exclusivité d'un régime sur les autres. En effet, remarque Barère : «Quand les pouvoirs sont bien indépendants, et quand ils ont la force et les lumières nécessaires, soit par leur organisation et leurs attributions, soit par les éléments et les hommes qui les composent, alors le pouvoir arrête le pouvoir, et il n'y a plus ni usurpation, ni puissance absolue, ni domination aristocratique, ni révolution démocratique à redouter » (32). La pierre de touche de ce système repose encore sur la Chambre des pairs, «magistrature nationale » qu'on ne saurait assimiler à une classe privilégiée, mais qui, au contraire, devient une expression de la

(30) Les Époques de la Nation française..., op. cit., p. 11.

(31) Id., pp. 19-21.

(32) Théorie de la Constitution...,op. cit., p. 3. 
"représentation héréditaire", placée ente le pouvoir du roi et le pouvoir des communes, à la condition du renouvellement fréquent de la Chambre de la nation et de la responsabilité des ministres, accusables par la Chambre des pairs. Enfin le vote exclusif des impôts revient à la Chambre des communes.

Ainsi défini, le problème du gouvernement fort ne met plus en scène le danger, par trop convenu, des abus du pouvoir exécutif, les risques rabâchés d'usurpation par le pouvoir exécutif des prérogatives des institutions législatives, dont beaucoup ont pu croire qu'ils étaient à l'origine des échecs successifs, des blocages institutionnels, en 1792, 1794, 1797, 1799, 1804, et 1815. Barère a justement l'intuition du contraire. «La non-exécution! Voilà le fléau qui s'est attaché aux Constitutions en France depuis 1791 jusqu'à 1814 [...] Aussi l'auteur immortel de L'Esprit des Lois dit, en parlant de Charlemagne : il fit d'admirables règlements; il fit plus, il les fit exécuter " (livre XXXI - chapitre 18), prouvant ainsi que l'exécution des Constitutions et des lois est encore plus difficile et plus importante que leur formation et leur promulgation. «Il est nécessaire [pour sortir de la crise permanente] que l'esprit d'exécution et la probité d'opinion animent également le prince et les ministres, les représentants de la nation et les divers fonctionnaires publics. Il faut que chacun d'eux se pénètre de cet esprit d'exécution qui est la religion de la loi » (33).

En quelques mots, déplaçant complètement l'axe traditionnel d'étude sur l'Exécutif, censé observer les règlements qui fixent la nature du pouvoir, et se pencher sur celui ou ceux qui en sont le ou les chef(s), l'auteur vient de faire progresser grandement la pensée du pouvoir exécutif. Sa proposition s'avère complètement différente dans la formulation des concepts d'« esprit d'exécution» et de "probité de l'opinion", qu'il fait reposer, en grande partie, sur les «divers fonctionnaires publics», donc à la base de l'État. Le pouvoir exécutif ne serait pas qu'une traduction effective de la loi; il est, à son origine et en son essence, différent. Peut-être même la loi n'est-elle que la traduction d'un pouvoir d'exécution qui paradoxalement la précède. Barère a l'intuition qu'un pouvoir se construit, en deçà des institutions, dans le lien qu'entretient l'administration avec l'opinion publique. Selon lui, en effet, au moment de l'exécution d'une loi qui, évidemment, au départ, se situe en amont de ces deux entités, il se crée entre ces dernières, sous forme de rapports autonomes, une sorte d'interaction - l'une agissant sur l'autre et réciproquement - qui sera à l'origine de nouvelles lois découlant d'un état de choses différent créé par l'application de la loi originelle.

En soi, le pouvoir exécutif ne semble rien d'autre que la faculté du gouvernant à créer de l'assentiment, d'où le constant souci dans un système 
dirigiste, du contrôle des mœurs et de l'instruction du peuple (34); d'où la préoccupation permanente dans un système libéral, du repérage des moindres signes de délégitimation de l'autorité ou de construction des oppositions utilisant différents médias. Dans les deux cas, l'encadrement de la société civile par des élus ou des fonctionnaires ne sert qu'à traduire en proposition de loi ou en exécution de la loi, un pouvoir d'exécution qui émane directement, mais selon des modalités différentes, des citoyens.

Peut-être, ce pouvoir exécutif, toujours personnalisé ou incarné, échappe-t-il à cet enfermement. Insaisissable, il repose lorsqu'il est bien construit, sur l'assentiment de tous, précédant la loi et lui succédant, composé d'éléments, $a$ priori, intrinsèquement différents, dont Barère perçoit la complexité d'agencement et la nécessité de les penser dans leur dynamique temporelle. Dès le début de son introduction, désormais plus claire, il avait affirmé, en fin connaisseur de l'Angleterre: «Cette Constitution, n'a pas cessé un instant, depuis plus d'un siècle, de produire l'extension coloniale et commerciale, d'appuyer la puissance maritime sur la domination des deux Indes, et de présenter, au sein des orages politiques qui couvraient l'Europe, le gouvernement le plus régulier et le plus fort, même sous un roi en démence et sous un régent soumis à des restrictions dans le pouvoir. La Constitution anglaise a donc une grande force intrinsèque, une grande puissance d'institution et d'organisation. Quelle en est la source?... la puissance et la souveraineté du peuple. Quels en sont les soutiens?... l'opinion publique, le jugement par jurés, la division exacte des pouvoirs, et surtout la liberté de la presse » (35).

Comment ces éléments de la société civile (la liberté de la presse) ou bien l'expression d'une forme politique si difficile à définir (l'opinion publique) peuvent-ils garantir l'excellence du pouvoir exécutif, voire son fondement? C'est ici qu'il faut revenir à l'Exécutif tel un esprit d'agir immédiatement, dans lequel l'esprit d'exécution et la probité sont déjà application de la loi. En soi, l'Exécutif n'est jamais l'expression d'une volonté personnelle ou d'un agir indépendant de l'agent exécuteur. Il n'est que l'expression d'une opinion libre et, ce qui effraie le plus le législateur, l'intervention la plus rapide possible; ceci pour le plus grand bonheur de qui commande et qui a su construire une relation spéculaire entre lui et le plus grand nombre, surtout lorsqu'il s'appelle Bonaparte (36).

(34) Voir l'article de Dominique Margairaz dans le présent numéro des A.H.R.F, et les réflexions de F. de Neufchâteau sur le temps nécessaire pour éduquer les citoyens.

(35) Théorie de la Constitution..., op. cit., p. 4.

(36) Bonaparte est le premier à utiliser cette vitesse, anticipant par exemple le résultat définitif du vote pour proclamer la Constitution de l'an VIII. De son côté, le Directoire poursuit la réflexion sur la vitesse de communication des informations politiques. Les pages que consacre la Décade à l'invention du télélogue, rouleau gigantesque placé devant une foule et percé de trous, derrière lesquels défilent les lettres qui finissent par composer un discours politique, en est une illustration (La Décade, 20 nivôse an VII, $\mathrm{n}^{\circ} 11, \mathrm{p} .122$ ). 


\section{Le Directoire exécutif, origine du pouvoir exécutif républicain}

Barère a, en grande partie, soulevé ces problèmes, en 1797, dans son cuvre De la Pensée du Gouvernement (37). Après la grande effervescence théorique du printemps et de l'été de l'an III, qui voit l'élaboration d'une nouvelle Constitution, l'an IV donne le jour à une autre saison théorique de réflexion sur le gouvernement représentatif, que ce soit du côté des démocrates, opposés au nouveau texte voté en fructidor an III et eux-mêmes en désaccord sur les modalités de la résistance, ou du côté des partisans de l'ordre, favorables à la nouvelle Constitution, mais opposés à leur tour sur l'interprétation des institutions (38). Parmi ces derniers, deux écrits se distinguent : le texte de Benjamin Constant, De la force du gouvernement actuel de la France et de la nécessité de s'y rallier, et celui de Lezay Marnésia, De la faiblesse du gouvernement et de la nécessité pour lui de gouverner selon le vou de la multitude (39). Pour ces deux défenseurs de l'ordre, la question posée par le Directoire, à ce moment précis de son existence, se résume en une réflexion sur le mode de fonctionnement, ainsi que sur la légitimité de son gouvernement.

Benjamin Constant démontre que, face aux périls qui ne cessent de le menacer sur sa droite et sa gauche, le gouvernement doit s'imposer en un centre qu'il contribue à édifier, à partir de présupposés que l'auteur expose dans la définition de ses opposants demeurés jacobins ou toujours royalistes. Ce premier constat oblige ceux qui sont en charge de la conduite de l'État, en cette période de risque de déstabilisation des institutions, à construire de façon autoritaire le pouvoir exécutif et à nommer de façon partiale des hommes dévoués aux principes républicains, qu'ils seront chargés d'exécuter de façon indépendante. L'objectif consiste à renforcer durablement la République, sans tenir compte d'une opinion publique, par nature versatile. « La justice est un devoir dans les gouvernants, l'impartialité serait une folie et un crime. Pour faire marcher une institution, il faut qu'un homme soit partial pour l'institution. Il ne faut pas que pyrrhonien politique, il aille recueillir les doutes, peser les probabilités et demander sans cesse à la majorité, si elle persiste à préférer la forme actuelle. L'esprit de l'homme est

(37) Sur les difficiles conditions de publication de l'ouvrage de Barère et les différentes éditions, nous renvoyons à l'article de Françoise BRUNEL, op. cit., pp. 323-324. Le Joumal de Paris, de Roederer, no 220, du 10 floréal an V, rend ainsi compte de l'élection de Barère : «C'est un scandale et même un malheur que la nomination de B. (sic) au corps législatif. II ne fera rien pour son parti mais il pourra servir de prétexte aux ennemis de la République [...]. Il est bon au reste, que l'on sache que l'artifice par lequel $B$. a capté les suffrages des bons habitants des Pyrénées, a été la dévotion. B est un dévot tout comme un autre; depuis un an c'est un petit saint ».

(38) Voir l'article de Christine Le Bozec dans le présent numéro des $A . H . R . F$.

(39) Benjamin CONSTANT, De la force du gouvernement actuel et de la nécessité de s'y rallier, Paris, 1796, réed. Champs Flammarion, préface et notes de Philippe Raynaud, Paris, 1988, et Adrien DE LEZAYMARNESIA, De la faiblesse du gouvemement et de la nécessité pour lui de gouverner selon le vau de la multitude, Paris, 1796. 
versatile. Il faut que les institutions soient stables. Il faut maintenir la majorité en la supposant invariable. Il faut lui rappeler ce qu'elle a voulu, lui apprendre ce qu'elle veut, en lui faisant trouver le bonheur et le repos sous les lois » (40). Pour un autre personnage qui souffre à son tour de la réputation d'un transfuge à tout va, - que n'a-t-on dit sur l'opportunisme de Constant - la fermeté semble de mise et l'effort de constance exercé par le pouvoir exécutif républicain, évident et sans contredit. Bien différente est la proposition de Lezay Marnésia. À son tour, le thème de la construction d'un centre politique motive son interrogation sur la forme la plus adéquate du gouvernement. Pour lui, en opposition radicale avec Benjamin Constant, « l'idée de la représentation des opinions et des intérêts doit être prise à la lettre : le gouvernement légitime sera celui qui exprime la majorité nationale, fondée sur le sentiment général et exprimée dans l'opinion publique » (41). L'Exécutif trouve sa raison d'être dans l'adéquation avec l'opinion qui s'exprime dans les suffrages et non dans un impératif supérieur de construction de la République, comme le soutient Constant.

Les deux systèmes placent le gouvernement, c'est-à-dire la conduite de l'Exécutif, au coeur d'une réflexion sur le sens et la nature de la volonté générale, soit pour montrer la nécessaire autonomie de la machine exécutante par rapport à l'opinion publique, dans le cas de Constant, soit son obligatoire soumission à l'esprit du plus grand nombre, selon Lezay (42). Dans cette perspective, un an plus tard, en l'an V, à l'approche des élections qui voient le parti d'une possible restauration de la monarchie constitutionnelle s'avancer vers les échéances électorales du printemps, en vainqueur potentiel, l'apport de Barère, est déterminant pour proposer un troisième type de relations entre le gouvernement et l'opinion publique. $\mathrm{Ni}$ autonome et coupé de la société civile, ni seule représentation servile d'une majorité conservatrice, le gouvernement construit une relation de réciprocité constante et évolutive avec l'opinion publique, par le jeu complexe et entremêlé des élections de représentants et de nomination de ses agents. Ce serait l'indépendance de l'opinion par rapport au gouvernement, - et non le contraire - exprimant en toute liberté sa position, voire son opposition, par le biais des convocations électorales qui garantiraient cette force, réciproque et partagée, entre l'Exécutif et l'opinion. Barère pense un Exécutif vraiment républicain, c'est-à-dire toujours responsable, toujours adéquat au projet d'édification commune du contrat social, et inscrit son projet dans une dynamique qui sera confirmée en l'an VI et en l'an VII.

(40) B. CONSTANT, De la force..., op. cit., p. 41.

(41) P. Raynaud, Préface de De la force du gouvemement..., op. cit., p. 15.

(42) P. Raynaud précise : «Pour Lezay, en cela étonnamment moderne, les buts du centre ne peuvent en fait être atteints que sous la condition paradoxale de sa faiblesse politique : c'est du jeu de la logique majoritaire dominée par "l'opinion", que doit venir la modération réciproque des deux partis antagonistes », op. cit., pp. 17-18. 
Dès l'introduction de La Pensée du Gouvernement, Barère reprend les catégories de John Locke, considérant le gouvernement civil comme un tout, dans lequel, la division prévaudrait et non la séparation des pouvoirs néfaste à la république (43). « J'entends par gouvernement dans toute sa latitude, le pouvoir qui fait la loi et le pouvoir qui l'exécute, c'est-à-dire, les deux conseils formant le corps législatif et le directoire exécutif de la République ». Dans cette conception, l'Exécutif devient « un centre de puissance [...], organisé, affermi, considéré, parce qu'il réunit la célérité à la force [...], l'énergie et la dignité des opérations extérieures, à la puissance bienfaisante des lois dans l'intérieur" (44). Plus loin, Barère précise sa conception : «Le gouvernement se compose de trois pensées, l'invention (proposer des lois), l'examen (adopter des lois), l'exécution pour diriger et accélérer l'exécution des lois fortement et uniformément dans toute la république... » (45). Peu après il développe son analyse : «J'appelle ici gouvernement, l'action première qui fait exécuter les lois, et qui dirige l'administration publique. Il se compose en France du directoire exécutif et des ministres [...] c'est dans l'agence suprême exécutive, que réside la pensée. J'ai voulu parler de la tête et non point des bras. J'appelle donc pensée du gouvernement la délibération du directoire, sur l'action des lois et sur l'esprit qui doit diriger leur exécution, dans l'ensemble de toutes les parties de la République ». Ainsi, Barère a bien soin de ne pas confondre police, administration générale et pouvoir coactif. L'enjeu est de taille car il consiste pour l'ancien membre du Comité de salut public à fonder l'action comme la conséquence d'une réflexion autonome du Directoire. L'application de la loi ne saurait se réduire à une exécution. Elle est, en son essence, une pensée qui devrait faire du pouvoir exécutif, l'égal du Législatif.

«Ainsi, ordonner et prévoir, exécuter et surveiller, faire agir et inspirer : voilà en peu de mots la théorie de gouvernement » (46). La succession d'infinitifs ne produit pas un effet de seule rhétorique pour ce maître de la langue française qu'est le citoyen de Tarbes, mais indique que le pouvoir exécutif est littéralement le verbe du gouvernement, celui qui donne son sens à sa politique. «Le directoire exécutif est dans la machine entière du gouvernement, ce que les lois du mouvement sont pour l'organisation de l'univers, ou bien pour rappeler une image familière, ce que le grand ressort est au mouvement mécanique d'une montre; les différents ministres en sont les rouages, les administrations départementales et les autorités constituées

(43) Cf. John LockE, Traité du gouvemement civil de sa véritable origine, de son étendue et de sa fin, chapitre XII, «Du Pouvoir législatif, exécutif et fédératif dans un État», Paris, réed. G.F., 1984, pp. 250-262, introduction de Simone Goyard-Fabre.

(44) BARÈrE, op. cit., "Préambule aux citoyens du département des Hautes-Pyrénées ».

(45) Id., La seconde partie de l'ouvrage complète la proposition « quant à l'exécution de la loi, (et) se compose de la police ou administration générale de l'intérieur, et des relations extérieures ».

(46) $I d$, p. 2 
n'en doivent être que les aiguilles qui marquent le bonheur du peuple". Homme de son temps, Barère décrit la grande et efficace construction que devrait être le gouvernement, "pour en faire une machine politique, un mécanisme social capable de produire l'ordre public et la prospérité de la nation » (47). Mais surtout, il semble, par la métaphore de la montre, que Barère insiste sur une dimension plus originale et essentiellement liée à la pratique : la mesure du temps comme aune de la qualité d'un pouvoir exécutif. Inscrire dans la durée un gouvernement demeure finalement, la seule façon de l'apprécier. C'est aussi pour cette raison que la définition du pouvoir exécutif relève moins de sa discussion théorique que de sa viabilité pratique dans la durée. Dès les premières pages de son ouvrage, Barère avait écrit, tel un gage de qualité : «Déjà cette Constitution vraiment républicaine [...] a triomphé pendant quinze mois, de toutes les attaques de l'étranger, de tous les complots machiavéliques, de tous les mouvements des factions» (48). Alors que le Directoire vient de surmonter le péril - en grande partie exagéré - causé par la Conspiration des Égaux, alors qu'il se trouve devant la menace d'une réactivation des réseaux contre-révolutionnaires, en 1797, Barère, rejeté dans la clandestinité, veut démontrer sa loyauté en affirmant pour son compte un des éléments que tous les acteurs du pouvoir exécutif ont dû éprouver depuis la crise de l'été 1791.

La construction d'un centre de commandement implique aussi qu'il existe conceptuellement, c'est-à-dire qu'il s'accompagne d'un programme qui lui permette de se définir par rapport à un jeu politique radicalement binaire. Du coup, Barère souligne la qualité de cet Exécutif qui préserve à la fois, de l'anarchie et du despotisme... argument typique du Directoire et responsable de la marginalisation des idéaux radicaux, mais que l'on aurait tort de lire comme la répétition d'une banalité de la décennie, comme un simple artifice rhétorique, masquant une carence intellectuelle de la part de tous les « honnêtes gens » qui soutiennent le Directoire exécutif, car il cache un fondement idéologique qui s'affirme au fur et à mesure que dure le régime. «Il faut donc un juste milieu pour asseoir un bon gouvernement républicain, vous ne le trouverez jamais dans les extrêmes » (49). La théorie du juste milieu (expression qui revient trois fois dans l'ensemble du texte), comme fondement idéologique du Directoire exécutif est posée. Barère conclut cette première série de définitions, n'hésitant pas à avouer une continuité révélatrice : «Tous les avantages qu'on peut retirer du gouverne-

(47) Termes employés par Barère dans ses Mémoires pour décrire la révision de la Constitution durant l'été 1791, op. cit., tome I, p. 329.

(48) BARÈRE, op. cit., “ Avertissement aux citoyens du département des Hautes-Pyrénées ».

(49) BARÈRE, De la pensée du gouvemement, op. cit., p. 3. 
ment monarchique, seront conquis pour le gouvernement républicain, et réunis avec tous les bienfaits de la liberté » $(50)$.

Enfin, le but est « de gouverner peu à mesure que l'on gouverne avec confiance". Cette conclusion, alors que ce qui précédait semblait au contraire, induire l'omniprésence de la mécanique gouvernementale au fondement de la politique, comme garantie même du bon fonctionnement des institutions, soulève au moins trois interrogations au moment de l'interpréter concrètement. Qu'est-ce que le «gouverner peu»? La théorie du gouvernement pour Barère implique-t-elle une pratique «invisible» du pouvoir exécutif, rendue possible par l'acceptation de son fondement et l'intégration par chacun des devoirs d'obéissance? Il y aurait là une forme d'unanimisme qui ne reflète en rien l'échiquier politique de 1797 et qui ne semble pas garantir l'expression libre de l'opposition ou de la résistance nécessaire à la loi arbitraire. Ou bien la réflexion de Barère implique-t-elle une pratique réellement continue, mais modérée? Le gouvernement « de la raison " fait référence à un centre modéré, tempéré par la connaissance partagée de droits et de devoirs, de la part des gouvernants et des gouvernés, ce qui paraît légitime, voire souhaitable, mais renvoie à toutes les apories de la décennie, lorsque ceux qui ont fait invariablement appel à la raison, n'ont pas manqué d'opprimer, dans la réalité des faits, de la façon la plus sévère, ceux qui refusaient de se plier à leur prétendu gouvernement de la raison. Ou bien encore, en dernier lieu, faut-il comprendre que le gouvernement fonctionne «peu » lorsqu'il agit de façon discontinue, par cycles, tel le ressort de l'horloge déjà mentionné, qu'il faut remonter régulièrement?

Barère ne tranche pas véritablement ou plutôt, il apporte un autre éclairage, en argumentant sur d'autres aspects du pouvoir exécutif, livrant ainsi quelques éléments de réponse qui permettent de réunir les trois questions. Lui-même pencherait pour une théorie du gouvernement permanent, animé d'une basse intensité qui assurerait dans le corps social les conditions d'une circulation constante de l'application de la loi. Un premier élément de réponse est suggéré par l'étude de la collégialité du Directoire, dans la Constitution de l'an III : "Se régénérant par époque fixe, le Directoire républicain peut exécuter sans obstacle et avec énergie, ce système suivi de bonheur constitutionnel et d'ordre public, qui manque toujours aux rois et à leurs ministres qui changent sans cesse [...] enfin le Directoire français peut, par sa seule organisation, trouver en lui-même cette opiniâtreté pour les utiles projets et pour les grandes améliorations, qui manque à la nation elle-même, soit par sa masse, soit par l'incohérence des diverses volontés

(50) Barère n'aura pas toujours cette appréciation laudative du Directoire. Rédigeant ses Mémoires sous la monarchie de Juillet, il revient sur la période, en évoquant « le gouvernement à cinq têtes, qui avait tous les vices de l'oligarchie, tout l'orgueil de l'aristocratie, toutes les violences de la démocratie, et pas un seul avantage de la monarchie constitutionnelle, la seule qui soit supportable et compatible avec les Lumières de ce siècle et le caractère de la nation française " (Mémoires, op. cit., tome III, p. 76). 
partielles, soit enfin par sa mobilité naturelle [...] C'est une idée neuve dans l'art social, c'est un progrès sensible des lumières publiques et du système représentatif» (51). Ce commentaire est d'autant plus important qu'en couplant la notion de fixité avec celle de régénération au cour de l'Exécutif, l'auteur rend plus explicite la notion de gouvernement représentatif. D'une certaine façon, Barère décrit les conditions de réussite du renouvellement annuel du Directoire qui, somme toute, de 1796 à 1799, a mieux fonctionné que le renouvellement chaotique du pouvoir législatif (52). Régulée de la sorte, la grande mobilité du gouvernement, caractère original cette fois, du pouvoir exécutif, aurait garanti une stabilité, confirmée au sommet de la construction politique, par ce principe de fixité et d'immobilité absolue qu'était la Constitution. Un problème se pose encore, et non des moindres aux yeux d'un Barère sensible, en ce sens, à tout le débat de l'an III. Quelle est la garantie proposée par son système face aux dérives toujours redoutables de l'Exécutif, une fois que celui-ci est installé et approuvé, et comment concevoir les rapports entre le gouvernement et l'opinion dans cette élaboration théorique?...

On sait que cette interrogation a fait l'objet d'un vaste débat dont Sieyès et Thibaudeau se sont montrés de pertinents penseurs. En clair, il s'agit d'inventer, d'imaginer dans la Constitution, un pouvoir au-dessus de la loi, capable de rendre, en cas de conflit, un arbitrage entre les différents pouvoirs. Ce pouvoir neutre est le plus souvent, pour les hommes de l'an III, à rechercher dans la formation d'un jury constitutionnaire ou bien dans la réalisation d'une Chambre supérieure, un sénat indépendant et aristocratique (53). On connaît les échecs de ces propositions, leurs conséquences jusque dans la dégradation du climat politique de l'an VII, lors de la révision de la Constitution, prônée par Sieyès (54). Se pose donc aux théoriciens du politique, le problème de la garantie des pouvoirs et pour les plus perspicaces, de la garantie de la garantie... et ce, à l'infini, comme le fait remarquer Thibaudeau. Barère, conscient de cet aspect essentiel du contrôle de l'Exécutif, soulève bien ce problème, mais retourne la proposition, en suggérant un autre lieu pour l'édification de ce pouvoir neutre et, paradoxalement, dans l'expression la plus partisane de la politique depuis le début de la Révolution... dans la presse et son entière liberté. "Trouvez un œil qui observe à tous les instants les législateurs, le Directoire, les ministres, les juges, les administrateurs, les magistrats du peuple et les commissaires du

(51) Barère, De la Pensée du gouvernement, op. cit., p. 9.

(52) Cf., après les travaux de Jean-René Suratteau et Isser Woloch, Bernard GaINOT, 1799, un nouveau jacobinisme?, Paris, Éd. du CTHS, 2001.

(53) Cf. Paolo Colombo, Governo e costituzione, op. cit., "parta terza, Cappitolo III "L'unité d'action" e il potere neutro : il dibattito sul direttorio », pp. 487-540.

(54) Voir “Observations constitutionnelles ", dans Des manuscrits de Sieyè 1773-1799, sous la direction de Christine FAURÉ, avec la collaboration de Jacques Guilhaumou, et Jacques Valier, pp. 517-527, Paris, Honoré Champion, 1999. 
gouvernement, trouvez un bras qui arrête, dénonce les abus de tout genre, les tyrannies, de toutes espèces, il n'en est qu'un, c'est la liberté de la presse...?»(55). L'auteur va encore plus loin lorsqu'il suggère que «la liberté de la presse provoque, à tous les instants, la réforme dans les lois et la justice dans le législateur, la correction dans les abus de l'administration et le patriotisme dans le gouvernement, l'exécution de la loi par les fonctionnaires publics et la modération dans les magistrats, l'effroi pour les dominateurs et la punition contre les ambitieux » (56).

Tout le vocabulaire des « honnêtes gens » qui ont pensé la Constitution de l'an III - modération des magistrats, réforme des abus, correction de l'arbitraire, et surtout régulation de l'opinion - se retrouve ici mais afin de suggérer une autre interprétation idéologique. La volonté de faire de la presse libre, non plus seulement une expression de la bonne marche de l'Exécutif, mais un des éléments constitutifs de ce même Exécutif s'impose. Alors que le débạt constitutionnel s'est construit, en grande partie, autour de la garantie de la liberté, ici, c'est la liberté de la garantie qui est revendiquée afin de ne pas tomber dans l'aporie infinie du cercle vicieux soulevé par Thibaudeau. Paradoxalement, mais «républicainement», la presse ne fonctionne pas comme l'illustration des libertés garanties; elle laisserait plutôt ce rôle au gouvernement. La presse fait fonctionner le pouvoir exécutif et non le contraire. «... Laissez faire et laissez imprimer, voilà toute la théorie du gouvernement sur la liberté de la presse et le régime des imprimeries... la contrebande des pensées fait tort au gouvernement et renverse la Constitution qui nécessite cette liberté ".

Dans ce système de libéralisme politique placé sous l'égide du gouvernement, liberté de la presse, liberté civile et liberté politique découlent les unes des autres. Ici Barère semble répondre à Constant pensant construire un gouvernement républicain hors d'une opinion érigée en objet de méfiance absolue parce qu'incontrôlable; et à Lezay voulant établir une simple instrumentalisation du gouvernement, reflet et agent servile d'une opinion versatile. "Cinq hommes chargés du gouvernement d'une population immense, divisée par une multitude d'opinions, et d'intérêts divers [...] trouveront dans la liberté de la presse [...] un grand levier d'opinion pour gouverner, un réverbère admirable pour éclairer la marche des fonctionnaires publics, et une reddition de compte journalier au peuple » (57). La force de l'Exécutif et du gouvernement naît d'une forme d'interaction entre société civile et société politique, ou plutôt, la presse traduit en termes politiques des aspects de la vie civile, pendant que les fonctionnaires imposent quotidiennement les décrets politiques. «C'est cette circulation égale et constante que le gouvernement doit

(55) Barère, De la Pensée du gouvernement, op. cit., p. 70.

(56) Id., p. 74.

(57) Id., p. 76. 
faciliter, activer et entretenir avec une surveillance journalière, c'est cette action du pouvoir constitué et cette réaction de l'opinion et des moyens du peuple qu'il importe le plus à l'administration générale de ne jamais laisser paralyser ou retarder, car si les principes d'économie, de législation, de police et d'ordre, descendent du gouvernement au peuple, les principes de vie, de force d'opinion et de richesse, montent du peuple au gouvernement » (58).

Soucieux de pragmatisme, l'auteur ne saurait s'arrêter à une description des relations entre le gouvernement et la presse, et aborde là l'aspect le plus détaillé de sa démonstration : l'ensemble du gouvernement ne repose en somme que sur la qualité de l'administration départementale, «le maillon le plus fort de la chaîne du ministère de l'Intérieur ». Le pouvoir exécutif construit ainsi une relation spéculaire avec le pouvoir législatif, sous le contrôle de l'opinion : la nomination des commissaires et des agents du Directoire, près le pouvoir exécutif dans chaque département, à laquelle est consacré le chapitre $\mathrm{XXV}$, doit répondre à l'élection des députés, et revêt, en bonne logique républicaine, la même importance. Le premier incarne les nécessités centralisatrices et nationales d'un pouvoir exécutif, le second les intérêts centrifuges et fédératifs d'une volonté locale, tous réunis et approuvés dans un gouvernement républicain par le jeu de l'opinion (59).

Reste un dernier problème et non des moindres: celui des rapports entre le pouvoir exécutif soucieux de stabilité et l'opinion publique telle qu'elle apparaît aux contemporains, dans son incontrôlable dangerosité depuis 1789. On l'a saisi, la référence constante au temps (durée d'un gouvernement, célérité de l'application de ses décisions) et aux modalités de circulation des informations et des ordres dans l'espace, fait de la maîtrise de la médiation, la condition de réussite de tout gouvernement, quel qu'il soit. Par-delà les institutions ou les administrations, Barère constate que le paradoxe du gouvernement républicain est de reposer sur un média qui lui échappe, sur une force sur laquelle il a peu de prise : l'esprit public ou opinion publique, ainsi définis par l'auteur (60). Il existe un esprit public

(58) $I d$. p. 53.

(59) « Ces fonctionnaires ont une influence directe et active sur l'administration départementale et municipale, sur la distribution de la justice et de la police; ils favorisent ou contrarient tous les éléments de l'agriculture, de l'industrie et du commerce, c'est-à-dire le rétablissement de la propriété des colonies, la tranquillité publique, la sûreté des personnes, le respect des propriétés, le salaire des travaux, l'équitable répartition des contributions, l'exécution de toutes les lois, la tolérance de toutes les opinions religieuses et politiques. Il importe donc que le Directoire fasse de bons choix, des choix de citoyens sages et éclairés, d'hommes probes, fermes et patriotes, pour les importantes fonctions des commissaires et des agents du pouvoir exécutif ", (id., p. 134)... ou lorsque Barère retrouve la logique de son vieux projet d'édification d'un système fédératif, d'une république fédérative, celle des départements-unis, dont le centre administratif se tiendrait à Paris, et le pouvoir législatif composé d'une chambre des représentants et d'une chambre fédérale, demeurerait itinérant, Mémoires, op. cit., «Plan d'une République fédérale », p. 39.

(60) Sergio Luzzatto a rappelé l'acuité du débat, en l'an III, à propos des distinctions entre deux notions proches, opinion publique et opinion du peuple, (L'automne de la Révolution, Paris, Honoré Champion, 2002 - 1re édition, en italien, 1994). 
sujet aux plus violentes variations, à des altérations, à des formes de versatilité qui risquent de fragiliser le gouvernement. Cette opinion est formée par les factions, essentiellement dans les grandes villes. Elles possèdent de puissants moyens d'expression, accaparent le débat politique et feraient croire à une radicalisation constante de l'opinion de tous. Ces «irascibles», ces «fanatiques », ces «vindicatifs de la royauté et de la république » - ce sont les termes de Barère - forment un écran entre le peuple et le gouvernement, usurpant le titre de représentants de l'opinion publique. Mais il existe aussi pour le bon administrateur, une autre opinion, authentique celle-là, que le gouvernement républicain doit savoir conquérir et gagner à sa cause. Cette opinion se caractérise, non par sa mobilité, sa versatilité ou ses écarts, mais bien au contraire par son inertie et son immobilisme.

Subtil sociologue politique, Barère constate : «C'est à la masse de la population et à la difficulté de la remuer, de la rallier à un mouvement préparée par des ambitieux ou des vindicatifs, que nous devons la mort ou l'abattement des factions, bien plus qu'à notre vertu publique, ou même à la force du gouvernement actuel; voilà un des grands avantages attachés à la vaste étendue des républiques » (61). Observateur très fin, l'auteur décrit les aspirations profondes de cette déjà majorité silencieuse et pourtant politisée que le gouvernement doit comprendre. Poursuivant le débat entre Constant et Marnésia, sans les nommer, Barère intitule son vingt-huitième chapitre, «De l'Intérêt commun et individuel de se rallier au Gouvernement, pour le Maintien de la Constitution républicaine de l'an III ». L'intérêt bien compris des individus et de la masse doit guider un ralliement à la République, signifiant la fin de la Révolution que tous désirent. «Ainsi obéir aux lois, non par la force, ni par la nécessité mais par affection ou du moins par la raison, c'est travailler à terminer la Révolution, à respirer à l'ombre des lois et sous l'empire de la cité [...] Le peuple ne peut obtenir des lois républicaines que le repos honorable qui lui convient, et ce que les lois forment de stable et de rassurant, il ne peut le recevoir que de la Constitution et du gouvernement qui la met en activité. Le peuple est donc toujours de fait et d'intérêt pour le gouvernement. Il y a plus, le peuple est pour le gouvernement, par cela même que c'est son ouvrage et sa volonté. Que doit-ce donc être, quand le gouvernement est son premier besoin? "(62). Toute une maturité politique se dévoile dans ses lignes. L'appareil exécutif doit créer les conditions d'un opportunisme de masse et d'une acceptation définitive et raisonnée de la République, en garantissant à ce plus grand nombre qui ne s'exprime jamais, repos et assurance de la sûreté personnelle.

(61) Id., p. 141, chapitre «Des Débris des Factions». Ces propos auraient pu et dû couper court à tous les commentaires désabusés de toutes les générations d'historiens se succédant et répétant à satiété l'indifférence du peuple vis-à-vis de la République, et l'échec programmé du Directoire, après 1795, devant la soi-disante apathie du peuple... Las, peu d'entre eux ont lu ou ont saisi la modernité de ces propos.

(62) BARÈRE, De la pensée du gouvernement, op. cit., p. 153. 
C'est pour cette raison encore que le gouvernement, s'appuyant sur la «modération [...] vertu des régimes constitutionnels » n'a aucune opinion, ou plutôt, " qu'il n'incline jamais vers aucune opinion, autre que celle de la franche république » (63). De fait, le gouvernement ne s'appuie jamais sur une faction pour la neutraliser, comme dans le système de Constant, ou ne représente jamais une faction, comme dans le système de Lezay Marnésia risquant d'être neutralisé. Littéralement le gouvernement n'a pas d'opinion, il est républicain et construit les modalités d'exécution de la Constitution de façon a-politique. Ce n'est pas à lui de créer un équilibre entre les partis, mais «c'est à l'opinion publique, à l'opinion nationale, à les balancer ellemême, à les affaiblir, à les ployer ou à aider le gouvernement pour les contenir ou les vaincre ". Là où Constant pensait un parti élitaire du centre, formé de l'agrégat des personnalités politiques ralliées, Barère lui oppose un gouvernement modéré et central, représentant la plus grande majorité des citoyens et, de la sorte, contraint de construire une politique centriste, sans s'interdire évidemment de mettre progressivement en place des institutions républicaines, telle l'éducation nationale, destinées à ancrer auprès des masses anonymes les bienfaits de la République. «N'est-ce donc rien que d'offrir à une vieille nation le spectacle d'un gouvernement libre représentatif-électif, fondé sur les mœurs et les lois, sur le travail et le patriotisme, sur la propriété et l'égalité ?»(64).

Au terme de cette lecture partielle de l'œuvre de Barère, quelques aspects de sa conception du pouvoir exécutif apparaissent plus clairement (65). Il n'est certainement pas une girouette, comme le voudraient un examen superficiel de sa trajectoire politique ou, plus encore, l'opinion de ses détracteurs, loin s'en faut; c'est un opportuniste de l'Exécutif, dans la mesure où il ne conçoit pas la vie politique comme l'échange des idées contraires et contradictoires, au sommet d'une structure parlementaire, mais comme la consolidation permanente du gouvernement républicain par les médiateurs (presse, administration) qui unissent la masse inerte mais sûrement pas amorphe des citoyens, à la grande activité des agents du pouvoir exécutif. Réaliste encore plus qu'opportuniste, Barère insiste : «Il y a trois choses dont un gouvernement sage ne doit jamais parler, mais sur lesquelles il doit toujours agir dans le silence; ce sont la religion, la police et les subsistances [...] Chacun veut être assuré de sa subsistance, ne pas trembler pour sa fortune, jouir de sa sûreté personnelle, et être libre pour sa croyance et son culte quels qu'ils soient » (66). Tout le pari revient à faire en

(63) Id, p. 145

(64) Id. p. 147.

(65) Bien des aspects de la pensée de Barère mériteraient de plus amples développements, à commencer par ses idées sur l'armée républicaine et sur la politique extérieure de la République.

(66) BARÈRE, De la pensée du gouvemement, op. cit., p. 133. 
sorte que ces objets, en soi a-politiques, constituent de forts symboles, et défendus, octroyés, garantis par la République, deviennent des marqueurs identitaires du Directoire, en 1797. La perspicacité de l'observateur politique est de mesurer et de comprendre la place que tiennent la crise économique, le discours sécuritaire déjà, et l'absence de régularisation des rapports de l'Église et de l'État, dans le discrédit du gouvernement et d'esquisser par là même la tâche du suivant. Autant de «conseils» que Bonaparte ne manquera pas d'exécuter, trois ans plus tard.

Pour n'avoir pas su créer cette police de l'ordre public, ni pu mettre en place cette neutralité idéologique au cœur de la politique du Directoire exécutif, pour n'avoir pas su créer les conditions d'un ralliement opportuniste et de masse autour d'un programme modéré et centriste, le gouvernement issu de la Constitution de l'an III, n'a pu se donner les moyens de sa perpétuation. Dans ces conditions, l'analyse de Barère sur une relation spéculaire entre le gouvernement et le peuple au fondement de la république demeure lettre morte. Le Consulat, au contraire, république enfin constituée et se voulant modérée - toujours en comparaison des vicissitudes causées par les «septembriseurs » ou les partisans des Bourbons - luttant à son tour contre les factions, mais se tenant aussi à l'écoute attentive de l'opinion par l'intermédiaire de son chef, grand communiquant s'il en est, en même temps que créateur d'autolégitimité par plébiscites interposés, avait créé les conditions du ralliement de nombreux anciens jacobins, conventionnels et personnels du gouvernement révolutionnaire. Un tel ralliement, dans de telles conditions, non seulement ne relevait pas de la pure palinodie politique, mais exprimait une forme de logique pour tous ceux qui n'avaient pas saisi dans le Directoire la nouveauté politique du combat des partis et de la conquête légale du pouvoir. Ces acteurs politiques considéraient ce régime comme une expérience gâchée de construction de la république du juste milieu (67)... Quant à Barère, retrouvant concrètement une liberté, habilement accordée par le Premier Consul, il ne pouvait être le dernier à se rallier.

Bien mieux que ne l'avait fait le Directoire, Bonaparte allait construire une République qui permettrait et encouragerait l'adhésion organisée autour d'une politique de l'ordre, représentée par la prédominance du pouvoir exécutif, renforçant le mépris du débat idéologique, dans l'édification d'un «extrême centre » républicain, fait de bon sens, d'honnêtes sentiments et de désirs de tranquillité, dans la construction d'un État policier et républicain. Depuis l'an V, Barère avait élaboré une première forme de cet

(67) Encore plus spectaculaire est l'attitude des patriotes italiens étudiés par Antonio DE FrancesCo, Vincenzo Cuoco, una vita politica, Roma, Laterza, 1997. 
opportunisme en affirmant : "J'ai été révolutionnaire; je suis constitutionnel. Ces deux états, loin de se contrarier, se défendent et s'appuient ». Or, reconnaître le texte de l'an III, c'était reconnaître que «la Constitution n'est placée dans aucun extrême; elle tient le juste milieu, elle est dans l'habitude facile de l'exécution et de l'obéissance; elle frappe dans toute la force du gouvernement légal et de toute la puissance d'un peuple libre. Elle est obéie de tous, elle commande à toutes les distances comme volonté nationale " (68). C'est du moins ce que Barère souhaitait ou faisait semblant de croire, cherchant le moyen, en ce début d'année 1797, de préparer son élection puis de la faire valider.

On ne saurait mieux réunir en quelques mots cette pensée originale du gouvernement républicain, en liant par une observation minutieuse des mécanismes de fonctionnement et de légitimation, trois termes, opportunisme, pouvoir exécutif, et " extrême centre ", qui semblaient hétérogènes $a$ priori et que la réflexion de Barère, éclairant son parcours et peut-être aussi celui de l'écrasante majorité des Français de 1795 à 1815, a contribué à penser ensemble. Silence ne vaut pas consentement, ralliement n'équivaut pas à trahison et modération ne renvoie pas à mansuétude... L'homme d'État que fut Barère, constant représentant du petit peuple de Bigorre, le savait bien.

Ainsi analysés par ce médiateur accompli, la république du juste milieu, mais non modérément républicaine, la politique du peuple et le pouvoir exécutif qui émane de lui, se révèlent souvent plus efficaces et autrement plus redoutables qu'on ne l'imagine...

Pierre SERNA

Université Paris I Panthéon-Sorbonne Institut d'histoire de la Révolution française

64, avenue du capitaine Jean 45800 Saint-Jean-de-Braye 